\section{Bukkales Fentanyl versus Morphin}

Mit bukkal verabreichten Fentanyltabletbesser lindern als mit oral appliziertem Morphin, wie aus den Ergebnissen einer italienischen Studie hervorgeht. In der Studie wurden Krebspatienten, die unter einer analgetischen Basistherapie standen, angehalten, dann um eine Medikation gegen Durchbruchschmerzen zu bitten, wenn die Schmerzen stärker wurden. Über drei Tage erhielten 68 Patienten bukkales Fentanyl $(100 \mu \mathrm{g})$ oder orales Morphin (10 mg) im Cross-over-Design.

Durch beide Präparate wurde die Schmerzintensität signifikant reduziert ( $\mathrm{p}$ $<0,0005)$. Eine signifikante Differenz zwischen den beiden Präparaten war jeweils 15 und 30 Minuten nach der Applikation ten lassen sich Durchbruchschmerzen zu beobachten. So wurde eine Schmerzreduktion um mindestens $33 \%$ (moderat) unter Fentanyl bei deutlich mehr der über 260 dokumentierten Schmerzepisoden registriert als unter dem Vergleichspräparat (nach 15 Minuten: 76,5 vs. 32,8\%; nach 30 Minuten: 89 vs. $54,9 \%$; p < 0,0005). Ebenfalls signifikant waren die Unterschiede, wenn eine Schmerzreduktion um mindestens $50 \%$ (substanziell) betrachtet wurde (nach 15 Minuten: 52,3 vs. 11,4\%; nach 30 Minuten: 75 vs. $45,8 \%$ ).

Unerwünschte Wirkungen wurden unbei am stärksten Übelkeit und Erbrechen 30 Minuten nach der Einnahme auftraten. Die Nebenwirkungen waren aber bei keinem Patienten schwerwiegend. ter beiden Medikationen beobachtet, wo-

\section{Nach gescheiterter Ersttherapie}

\section{Alternatives Kombinationsregime bei CLL}

Entwickeln Patienten mit einer CLL ein Rezidiv oder sprechen sie auf die Ersttherapie nicht mehr an, bieten hochdosierte Kortikosteroide eine wichtige Alternative für die Weiterbehandlung. Die Kombination mit einem alternativen Anti-CD20Antikörper kann das Ansprechen und Gesamtüberleben verbessern, wie eine aktuelle Studie zeigt.

Hochdosiertes Methylprednisolon (HDMP) oder Dexamethason in Kombination mit Rituximab zeigt Aktivität bei der rezidivierten bzw. therapieresistenten CLL, allerdings erkauft mit einer hohen Inzidenz schwerer Infektionen und nur kurzen progressionsfreien Krankheitsintervallen. Dexamethason scheint weniger nebenwirkungsträchtig als HDMP, übt zudem synergistisch direkte apoptotische sowie antiproliferative Effekte auf die Lymphom-Zelllinien aus und erhöht die Sensitivität für Rituximab.

Der humane monoklonale IgG1-Antikörper Ofatumumab bindet an ein anderes Epitop auf dem Rezeptorprotein CD20 als Rituximab und erreicht eine höhere Oberflächendichte, eine effizientere komplementabhängige Zytotoxizität und eine höhere antikörperabhängige zellvermittelte Zytotoxizität auch bei geringer CD20-Expression.

Vor diesem Hintergrund entwarfen Wissenschaftler um Michael Doubek eine Phase-II-Studie zur Evaluierung des Effekts einer Ofatumumab-DexamethasonKombination (O-Dex) auf das Gesamtanprechen von Patienten mit einer rezidivierten/therapieresistenten CLL. 33 Patienten durchliefen das O-Dex-Regime in geplanten sechs Zyklen. Daneben erhielten die Patienten oral 40 mg Dexamethason an den Tagen 1-4 und 15-18.

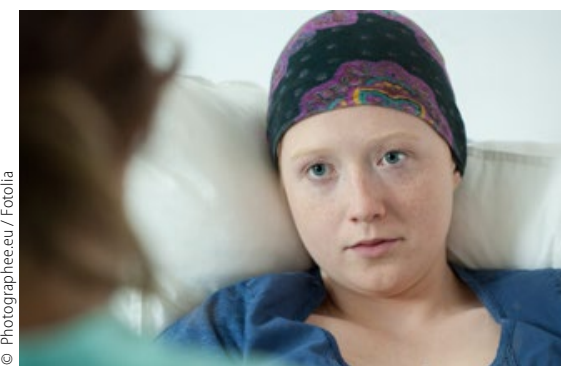

Bei Durchbruchschmerzen ist rasche Hilfe gefragt.

Den Unterschied zwischen den Wirksamkeiten der beiden Präparate führen Mercadante und seine Kollegen im Wesentlichen auf die unterschiedlichen Pharmakokinetiken zurück. Peter Leiner

Mercadante S et al. Fentanyl Buccal Tablet vs. Oral Morphine in Doses Proportional to the Basal Opioid Regimen for the Management of Breakthrough Cancer Pain: A Randomized, Crossover, Comparison Study. J Pain Symptom Manage. 2015 Nov;50(5):579-86.

$67 \%$ der Patienten erreichten ein komplettes Ansprechen und 15\% eine komplette Remission. Das mediane progressionsfreie Überleben betrug zehn, das mediane Gesamtüberleben 34 Monate. $33 \%$ der Patienten entwickelten höhergradige Infektionen. Im Vergleich zu publizierten Rituximab-Dexamethason-Regimes waren die Ansprech- und Remissionsraten unter der Ofatumumab/Dexamethason relativ hoch bei einem günstigeren progressionsfreien und $\mathrm{Ge}$ samtüberleben. Wolfgang Zimmermann

Doubek $M$ et al. Ofatumumab added to dexamethasone in patients with relapsed or refractory chronic lymphocytic leukemia: Results from a phase II study. Am J Hematol. 2015;90(5):417-21.

\section{kurz notiert}

\section{Cathepsin K unterstützt Krebszellen beim Einwandern in Knochengewebe}

Krebszellen, die ins Knochengewebe wandern, beginnen, das Protein Cathepsin K zu produzieren. Damit steigern sie ihre Überlebensfähigkeit in der Umgebung des Knochens, wie Freiburger Wissenschaftler nun herausgefunden haben. In ihren Untersuchungen stellten sie fest, dass Cathepsin K die Matrix-Metalloproteinase 9 (MMP-9) unter sauren Bedingungen, wie sie in Tumoren und während der Knochenresorption vorherrschen, spalten und aktivieren kann. Die Aufgaben der MMP-9 sind der Abbau und das Remodeling der extrazelluläre Matrix. Damit wird den Krebszellen ermöglicht, sich im Knochen einzunisten. Außerdem aktiviert MMP-9 Proteine, die die Bildung neuer Blutgefäße fördern. Damit wurde ein wichtiger, bisher unbekannter Zusammenhang zwischen der Expression von Cathep$\sin \mathrm{K}$ in Tumoren und Knochen und dem Remodeling der extrazellulären Matrix durch die MMP-9Aktivierung entdeckt [Christensen J et al. BMC Research Notes 2015;8:322].

Judith Neumaie 